\title{
Was kann das Konzept der Diskriminierung für die Medizinethik leisten? - Eine Analyse
}

\author{
Maximiliane Hädicke • Claudia Wiesemann (DD
}

Eingegangen: 10. November 2020 / Angenommen: 25. März 2021 / Online publiziert: 28. April 2021 (C) Der/die Autor(en) 2021

Zusammenfassung Kaum ein Begriff der ethischen Debatten der letzten Jahre hat eine solche Konjunktur erlebt wie der Begriff der Diskriminierung. Eine vergleichende Erörterung des Konzepts einschließlich seiner begrifflichen Nuancen und seiner ethischen Bedeutung für das Gesundheitswesen fehlte jedoch bislang. Ziel dieses Beitrags ist die Entwicklung eines differenzierten Verständnisses von Diskriminierung auf der Basis der philosophischen und soziologischen Literatur vor dem Hintergrund ethisch relevanter medizinischer und pflegerischer Szenarios. Anhand von praktischen Beispielen aus dem Gesundheitswesen erörtern wir die Besonderheiten direkter, indirekter und statistischer Diskriminierung, wir untersuchen die Bedeutung von Absichten und die Rolle von kollektiven Akteuren. Zudem diskutieren wir, welche sozialen Gruppen diskriminiert werden können und welche Rolle Formen epistemischer Ungerechtigkeit dabei spielen. Argumentiert wird, dass das Konzept trotz seiner Komplexität einen signifikanten Mehrwert bietet, weil es erlaubt, Formen der Benachteiligung aufzuzeigen, die über die Verletzung einzelner spezifischer Rechte oder Interessen hinausgehen. Als wissensbasiertes System von großem gesellschaftlichen Einfluss weist das Gesundheitswesen einige Eigenschaften auf, die Diskriminierung begünstigen können. Dies zu erkennen ist unerlässlich, um Diskriminierung wirksam entgegentreten zu können.

Schlüsselwörter Diskriminierung · Sozial saliente Gruppe · Gesundheitswesen • Soziologie $\cdot$ Epistemische Ungerechtigkeit

M. Hädicke $(\bowtie) \cdot$ Prof. Dr. C. Wiesemann

Institut für Ethik und Geschichte der Medizin, Universitätsmedizin Göttingen,

Humboldtallee 36, 37073 Göttingen, Deutschland

E-Mail: maximiliane.haedicke@med.uni-goettingen.de 


\title{
What can the concept of discrimination contribute to medical ethics?-An analysis
}

\begin{abstract}
Definition of the problem Few concepts in recent ethical debates have enjoyed as much popularity as the concept of discrimination. However, a comparative discussion of the concept, including its conceptual nuances and its ethical significance for health care, has so far been lacking. The aim of this paper is to develop a nuanced understanding of discrimination based on the philosophical and sociological literature against the background of ethically relevant medical and nursing scenarios. Methods Using practical examples from health care, we discuss direct, indirect, and statistical discrimination; we examine the importance of intentions and the role of collective actors. In addition, we analyze which social groups may experience discrimination and the respective importance of forms of epistemic injustice.

Conclusions We argue that despite its complexity, the concept offers significant added value because it allows us to highlight forms of disadvantage that go beyond the violation of individual specific rights or interests. As a knowledge-based system of great societal influence, health care exhibits some characteristics that can foster discrimination. Acknowledging this fact is essential in order to effectively address discrimination.
\end{abstract}

Keywords Discrimination · Socially salient group $\cdot$ Health care $\cdot$ Sociology · Epistemic injustice

\section{Diskriminierung als Gegenstand der Medizinethik - eine Einleitung}

Diskriminierung ist ein in der Medizin- und Bioethik oft verwendetes Konzept. Die UNESCO Universal Declaration on Bioethics and Human Rights hält in Artikel 11 fest: „No individual or group should be discriminated against or stigmatized on any grounds, in violation of human dignity, human rights and fundamental freedoms“ (UNESCO 2005). Derzeit diskutiert etwa die US-amerikanische Bioethik unter dem Stichwort „Black Bioethics“ intensiv darüber, ob und auf welche Weise nicht nur das Gesundheitswesen, sondern auch die Bioethik als Profession People of Color systematisch diskriminiert und wie sich das verhindern ließe (vgl. Ray 2020).

Umso wichtiger ist es, die Bedeutung und Reichweite dieses philosophisch anspruchsvollen Begriffs möglichst präzise zu erfassen. Dies geschah bisher in einer Reihe von Einzelstudien. In der ethischen Fachwelt wird etwa seit längerem das Thema der genetischen Diskriminierung diskutiert (vgl. Rehmann-Sutter 2005; Lemke und Liebsch 2015), und die Frage, ob Menschen mit Behinderung durch Pränatal- und Präimplantationsdiagnostik diskriminiert werden, sorgt schon seit etlichen Jahren für kontrovers geführte Debatten (vgl. Gen-ethisches Netzwerk e. V. 2014; Graumann und Koopmann 2018; Merkel 2019). Auch in den Arbeiten zur Menschenwürde wird auf Diskriminierung und ihre moralische Verwerflichkeit Bezug genommen (vgl. Stoecker 2011; Schaber 2012). In der Public-Health-Ethik, etwa im Themenfeld der Gesundheitskommunikation (vgl. Loss und Nagel 2009), wird Dis- 
kriminierung als wichtige Herausforderung diskutiert, und Florian Steger bündelte bereits 2007 in einem Sammelband Beiträge zu Stigmatisierung und Diskriminierung in Psychotherapie und Sexualwissenschaft. Dabei liegt der Fokus in der Regel auf den konkreten Auswirkungen im jeweils untersuchten Feld. Eine Erörterung des Konzepts der Diskriminierung in seiner themen- und kontextübergreifenden ethischen Bedeutung für das Gesundheitswesen fehlte bislang. Der vorliegende Beitrag will eine solche Analyse vornehmen.

Worum geht es genau, wenn von Diskriminierung im Gesundheitswesen gesprochen wird? Diskriminierung lässt sich, so unser Ausgangspunkt, nicht nur als eine individuelle Verletzung von Interessen oder Rechten verstehen, sondern als ein Phänomen, das in gesellschaftliche Zusammenhänge einbettet ist und dadurch eigene normative Fragen aufwirft. Es ist in der Lage, das Leben von Menschen aufgrund ihrer Gruppenzugehörigkeit(en) lebensbereichsübergreifend zu beeinträchtigen und kann unterschiedliche Ausprägungen wie etwa Rassismus, Sexismus, Ableism oder Ageism annehmen.

Angesichts der Vielfalt der Verwendungszusammenhänge scheint es angebracht, sich über ein ausreichend differenziertes Verständnis des Begriffs zu verständigen und damit eine Grundlage für weiterführende Diskussionen zu schaffen. $\mathrm{Zu}$ diesem Zweck werden wir die relevante philosophische Literatur vorstellen und im Licht von Beispielen aus dem medizinischen und pflegerischen Kontext diskutieren. Ziel dieses Beitrags ist es, die philosophische Debatte so aufzuarbeiten, dass sie für medizin- und bioethische Debatten zuträglich ist.

Hierfür haben wir den Beitrag wie folgt strukturiert. Beginnen werden wir mit einer Darstellung prominenter philosophischer Positionen zu der Frage, was moralisch falsch daran ist, wenn diskriminiert wird. Danach diskutieren wir die direkte und indirekte Diskriminierung und fragen, ob es einer Absicht bedarf, um zu diskriminieren. Schließlich gehen wir in diesem Kapitel auf das Konzept der statistischen Diskriminierung ein, welche in philosophischen Analysen als Sonderfall diskutiert wird und im medizinischen Kontext aufgrund der häufigen Verwendung von statistischen Daten nicht aus dem Blick geraten sollte. Entlang der Ideen der organisationalen Diskriminierung werden wir beleuchten, welche ethisch relevanten Erkenntnisse daraus gewonnen werden können, kollektive Akteure gesondert in den Blick zu nehmen. Diese Unterscheidung ist für das Gesundheitswesen besonders relevant, weil sich die handelnden Individuen in einem stark von Regeln vorstrukturierten System bewegen. Im Anschluss daran kommen wir zu einer Kerndebatte: Wer kann diskriminiert werden? Wir stellen unterschiedliche Zugangsweisen zu dieser Frage vor und entwickeln eine für die anwendungsbezogenen Belange der Medizinund Bioethik praktikable Position. Schließlich untersuchen wir, welche Anregungen das Konzept der epistemischen Ungerechtigkeit für eine ethische Debatte über Diskriminierung in einem wissensbasierten System wie der Medizin bietet. In einem Schlusskapitel fassen wir die wesentlichen Erkenntnisse zusammen. 


\section{Moralische Relevanz}

In der philosophischen Literatur wird zwischen einem generischen und einem normativen Diskriminierungsbegriff unterschieden. Im generischen Sinne meint der Begriff eine wertneutrale Unterscheidung und dementsprechend eine zwar unterschiedliche, aber nicht wertende Behandlung von Menschen ${ }^{1}$. Wird er in einem normativen Sinne verwendet, so meint Diskriminierung ,differential treatment plus something else" (Lippert-Rasmussen 2018, S. 2) und wird mit Gleichheits- und Gerechtigkeitsvorstellungen in Verbindung gebracht. Sprechen wir im Folgenden von Diskriminierung, so verwenden wir den Begriff ausschließlich in seinem normativen, moralisch relevanten Sinn. Diese Verwendung des Begriffs ist im deutschsprachigen Raum nicht nur verbreiteter, sie ist auch der zentrale Ausgangspunkt für ethische Fachdiskussionen, denn eine der Kernfragen kreist um das ,something else“. Was ist es und wie lässt es sich begrifflich fassen? Oder anders: Was macht Diskriminierung moralisch falsch?

Abhängig von der zugrunde gelegten ethischen Theorie lassen sich verschiedene Interpretationen des durch Diskriminierung bewirkten Übels unterscheiden. Konsequentialistische Positionen stellen den von Diskriminierung verursachten Schaden (harm) (Lippert-Rasmussen 2006, 2013; Thomsen 2011, 2013, 2015) ins Zentrum. Nach Moreau (2010) ist Diskriminierung dagegen moralisch zu verurteilen, weil sie Freiheitsrechte verletzt (deliberative-freedoms account). Andere (vgl. Eidelson 2015; Hellman 2008) wiederum sehen das wesentliche Übel in der Missachtung $\left(\right.$ disrespect $^{2}$ ), die mit Diskriminierung zum Ausdruck gebracht wird und womit der moralische Status des Individuums abgewertet werde.

Betrachten wir hierzu ein Bespiel aus der Medizin. Noch bis 2017 sahen die Richtlinien der Bundesärztekammer (BÄK) vor, dass eine In-vitro-Fertilisation nur bei heterosexuellen Paaren durchgeführt werden dürfe (vgl. BÄK 2006). Dies wurde als Diskriminierung von lesbischen Paaren kritisiert. Doch welches moralische Übel erfährt ein lesbisches Paar mit Kinderwunsch in der skizzierten Situation? Vertreter:innen des harm-basierten Ansatzes fokussieren dabei auf die Folgen. Der Schaden kann darin gesehen werden, dass die Frauen kein biologisch eigenes Kind bekommen können. Moreau (2010) würde argumentieren, dass die Ungleichbehandlung moralisch falsch ist, weil sie Freiheiten einschränkt, auf welche die Betroffenen ein Recht haben; in diesem Fall ist das die Fortpflanzungsfreiheit bzw. das Recht, eine Familie zu gründen. So kritisieren etwa auch die Nationale Akademie der Wissenschaften und die Union der deutschen Akademien der Wissenschaften, dass Eingriffe in die Fortpflanzungsfreiheit generell ,,als in hohem Maße begründungsbedürftig“" (2019, S. 25) gelten und eine Ungleichbehandlung allein aufgrund des Geschlechts oder der sexuellen Orientierung nicht legitim sei (Nationale Akademie der Wissenschaften, Union der Akademien der Nationale Akademie der Wissenschaften und Union der Akademien der Wissenschaften 2019, S. 29). Vertreter:innen eines disrespect-Ansatzes würden dagegen die Herabsetzung des lesbischen Paars hervorheben: Diesen Frauen werde kommuniziert, dass ihre Lebensform einer he-

1 ,Discriminare“ (lat.) = unterscheiden.

2 Für eine Übersicht zu unterschiedlichen Interpretationen von disrespect (siehe Beeghly 2018, S. 86). 
terosexuellen Ehe nicht gleichranging und ihr Kinderwunsch nicht in der gleichen Weise unterstützenswert sei. Dadurch würden sie als Personen herabgewürdigt.

Während mit einem auf den unmittelbaren Schaden oder die Einschränkung der Freiheit ausgerichteten Ansatz der Fokus stärker auf die Folgen der Handlung gelegt wird, rückt ein disrespect-Ansatz die Art der Handlung in den Mittelpunkt (vgl. Boshammer 2008). Beide Ansätze wurden wiederum auch kritisiert. So bringen manche Autor:innen vor, dass nicht alle Fälle von Diskriminierung von diesen beiden Interpretationen erfasst werden können, und schlagen vor, das Unrecht in der Einschränkung der Chancengleichheit (vgl. Knight 2018) zu verorten. Vermittelnde Positionen argumentieren, dass durch Diskriminierung sowohl harm verursacht als auch disrespect zum Ausdruck gebracht werden kann (Scanlon 2008, S. 73). Die zugrunde liegende moralphilosophische Debatte kann an dieser Stelle nicht umfassend dargelegt werden. Für die praktischen Belange der Medizinethik scheint uns sinnvoll, alle denkbaren Schadenspotentiale von Diskriminierung in den Blick zu nehmen. Ein pluralistischer Ansatz ist dafür am ehesten angemessen. ${ }^{3}$ Ein solches Verständnis liegt daher den weiteren Ausführungen zu Grunde.

\section{Formen von Diskriminierung}

\section{Direkte und indirekte Diskriminierung}

In den meisten philosophischen Arbeiten werden direkte und indirekte Diskriminierung voneinander unterschieden. Von direkter Diskriminierung wird gesprochen, wenn eine Ungleichbehandlung unmittelbar auf ein diskriminierungsrelevantes Merkmal zurückgeführt werden kann (,disparate treatment“, Klonschinski 2020, S. 142). So schließt beispielsweise die Hämotherapierichtlinie der BÄK direkt und unmissverständlich Personen für ein Jahr von der Blutspende aus, „deren Sexualverhalten ein gegenüber der Allgemeinbevölkerung deutlich erhöhtes Übertragungsrisiko für durch Blut übertragbare schwere Infektionskrankheiten wie HBV, HCV oder HIV, birgt“ (BÄK 2017, S. 18). Dies gilt für heterosexuelle Männer und Frauen nur dann, wenn diese häufig wechselnde Partner:innen haben, jedoch für alle Männer, die mit Männern Sexualverkehr haben, ohne Berücksichtigung ihrer individuellen Lebensweise, somit auch etwa für ein monogam lebendes schwules Paar. Dieser pauschale Ausschluss von homosexuellen Männern wird als diskriminierend kritisiert (Antidiskriminierungsstelle des Bundes 2017, S. 280; Deutsche Aidshilfe 2020), weil damit das Sexualverhalten schwuler Männer ohne Unterschied als fahrlässig, risikohaft und somit negativ bewertet wird. Auf diese Weise werde die schwule Lebensweise und jeder homosexuelle Mann abgewertet.

Indirekte Diskriminierung erfolgt demgegenüber, wenn Unterscheidungen auf der Basis eines scheinbar neutralen Kriteriums zur Folge haben, dass die Interessen be-

\footnotetext{
3 Auch Klonschinski (2020, S. 139), geht davon aus, dass nur ein pluralistischer Ansatz erfolgversprechend ist. $\mathrm{Ob}$ ein solches plurales Begriffsverständnis die konkurrierenden Ansätze unter sich widerspruchsfrei verbinden kann, müsste allerdings weiter geprüft werden. In der philosophischen Fachdebatte besteht in dieser Hinsicht kein Konsens.
} 
stimmter Gruppen systematisch benachteiligt werden (vgl. Lippert-Rasmussen 2006, S. 170). Mit ihr werden also Verhaltensweisen und Regelungen erfasst, deren diskriminierender Gehalt erst in den mitunter gravierend unterschiedlichen Folgen für die betroffenen Individuen zu Tage tritt (,disparate impact“, Klonschinski 2020, S. 142). So wurde etwa aktuell kritisiert, die Empfehlungen zur Triage bei der Beatmung von Corona-Patient:innen (vgl. DIVI 2020) diskriminiere Menschen mit Behinderung, denn das dort vorgeschlagene Kriterium der Erfolgsaussicht sei nur scheinbar neutral. Tatsächlich stütze es sich auf eine Gebrechlichkeitsskala, bei deren Anwendung Menschen mit Behinderung pauschal schlechtere Chancen zugeteilt würden (vgl. Verein für Gleichstellung und Menschenrechte Behinderter e. V. (NW3) und Interessenvertretung Selbstbestimmt Leben in Deutschland e. V. (ISL) 2020). Auch beim Einsatz von Algorithmen in der Medizin kann es zu indirekter Diskriminierung aufgrund vermeintlich neutraler Kriterien kommen. So wies etwa eine in Science erschienene Studie nach, dass ein in US-amerikanischen Krankenhäusern häufig verwendeter Algorithmus die Behandlungsbedürftigkeit von People of Color systematisch als zu niedrig einschätzt, weil er Entscheidungen aufgrund des vermeintlich neutralen Kriteriums der in der Vergangenheit im Durchschnitt angefallenen Behandlungskosten trifft. Die niedrigeren Kosten sind aber nicht durch geringere medizinische Behandlungsbedürftigkeit begründet, sondern durch sozial bedingt schlechtere Behandlungschancen (Obermeyer et al. 2019).

\section{Diskriminierende Absichten}

Einer verbreiteten Ansicht zufolge wird direkte Diskriminierung mit einer Intention zu diskriminieren und indirekte Diskriminierung mit ihrer Abwesenheit in Verbindung gebracht (vgl. Boshammer 2008, Kap. 2.2). Dies ist naheliegend, denn indirekte Diskriminierung tritt insbesondere dann gehäuft auf, wenn die Interessen mancher Personengruppen systematisch unbedacht bleiben. Dass etwas unbedacht bleibt, impliziert bereits, dass dabei nicht zwingend eine Absicht erforderlich ist.

Untersuchungen, die in der Critical Philosophy of Race zu verorten sind, zeigen jedoch, dass die mangelnde Berücksichtigung zumindest im Lichte von Ignoranz oder Gleichgültigkeit zu diskutieren ist.

So werden etwa Abbildungen in dermatologischen Lehrbüchern aufgrund ihrer Anschaulichkeit ausgewählt. Dies kann als neutrales Unterscheidungskriterium bei der Bildauswahl gelten. Obwohl das Erscheinungsbild nicht weniger Hauterkrankungen bei People of Color abweichen kann, werden ganz überwiegend Bilder von Erkrankungen auf heller Haut publiziert. ${ }^{4}$ Die fehlende Darstellung auf dunkler Haut kann unzureichende Fachkenntnisse des medizinischen Personals, Fehldiagnosen und schlimmstenfalls dramatische Folgen für die Gesundheit von People of Color zur Folge haben. Die moralische Problematik liegt hier nicht in der Wahl des Kriteriums, denn das Kriterium der Anschaulichkeit ist neutral und sinnvoll. Sie entsteht, wenn es wenig differenziert und damit ignorant oder gleichgültig für die Belange von People of Color umgesetzt wird.

\footnotetext{
4 Die Initiative „Mind the Gap“ hat dies aufgegriffen und ein medizinisches Handbuch zum Erscheinungsbild von Hautkrankheiten auf dunklerer Haut online veröffentlicht (vgl. Mukwende et al. 2020).
} 
Lippert-Rasmussen (2006, S. 172) argumentiert darüber hinaus, dass der Zusammenhang zwischen Intentionen und direkter Diskriminierung ebenfalls weniger eindeutig als angenommen sei. Wie direkte Diskriminierung ohne eigene diskriminierende Absicht der handelnden Person möglich sein kann, bedarf weiterer Erläuterung. Dazu folgender Fall: In einer Privatklinik arbeitet kein ärztliches Personal mit Migrationshintergrund. Die zuständige Klinikleitung weist einen Diskriminierungsvorwurf mit der Begründung zurück, sie habe nur die Interessen der Patient:innen bedacht, diese würden sich nicht gern von ihnen fremd erscheinenden Personen behandeln lassen. Mit ihrem Verweis auf die Haltung Dritter will die Klinikleitung unterstreichen, dass sie selbst keine diskriminierende Absicht mit ihrer Einstellungspolitik verfolge. Für die Bestimmung der Form der Diskriminierung ist es, LippertRasmussen (2006, S. 172) folgend, jedoch nicht ausschlaggebend, mit welcher unmittelbaren Intention gehandelt wird, sondern welches Unterscheidungskriterium zugrunde gelegt wird. Ob die Klinikleitung selbst rassistische Absichten hat oder sich bei ihren Handlungen von den rassistischen Einstellungen Dritter leiten lässt, ist dabei unerheblich.

Weiterhin wird darauf verwiesen, dass Vorurteile und Stereotypen auch unbewusst wirken und Handlungen beeinflussen können (implicit bias). ${ }^{5}$ Im Gesundheitswesen wäre das etwa der Fall, wenn sich ein:e medizinische:r Fachangestellte:r bei der telefonischen Terminvereinbarung in der Regel stets freundlich und geduldig, gegenüber Personen mit starkem Akzent hingegen kurz angebunden und ungeduldig zeigt, weil sie Menschen, deren Muttersprache nicht Deutsch ist, unbewusst ablehnt.

Im formal-logischen Sinne muss eine reflektierte Intention also, so Lippert-Rasmussen (2006, S. 173), keine Voraussetzung für direkte Diskriminierung sein. Weiterhin wirft dies die brisante und rege diskutierte Frage danach auf, ob für Handlungen, die auf unbewussten Vorurteilen fußen, moralische Verantwortung übernommen werden kann oder muss (vgl. Brownstein 2019). Dabei werden unterschiedliche Formen der Verantwortungsübernahme unterschieden, auf welche hier nicht im Detail eingegangen werden kann. Diskutiert wird etwa, ob zumindest eine moralische Verantwortung bestehe, sich seiner Vorurteile bewusst zu werden und diese in der Zukunft möglichst abzustellen.

Wichtig ist festzuhalten, dass entgegen der verbreiteten Annahme, eine Gleichsetzung von direkter und indirekter Diskriminierung mit der An- oder Abwesenheit von Intentionen in vielen Fällen zu kurz greift. Intentionen sind ohnehin nicht ausschlaggebend dafür, ob eine Diskriminierung vorliegt, zumal die Gründe des Gegenübers für die betroffene Person selten ersichtlich sind und Unabsichtlichkeit den entstehenden Schaden nicht zwangsläufig schmälert.

Die Intentionen des oder der Handelnden sind für eine ethische Auseinandersetzung mit Diskriminierung in der Medizin jedoch relevant, um ein Kontinuum der Verantwortung bestimmen und moralische Schlussfolgerungen differenzieren zu können. Wird intentional diskriminiert, ist dies in jedem Fall zu verurteilen, während eine Person für eine unbeabsichtigte Handlung nicht in der gleichen Weise zur Rechenschaft gezogen werden kann. Praktisch relevant werden die Erkenntnis-

\footnotetext{
${ }^{5}$ Für eine systematische Literaturanalyse zur Thematik impliziter Vorurteile bei Personal im Gesundheitswesen siehe Fitzgerald und Hurst (2017).
} 
se insbesondere dann, wenn Maßnahmen entwickelt werden, die diskriminierenden Handlungen entgegenwirken sollen. Es macht einen gravierenden Unterschied aus, ob ein Veranstaltungsformat das Ziel verfolgt, nicht-intendierte diskriminierende Handlungen zu reflektieren, um sie in Zukunft besser vermeiden zu können, oder ob intentionale und reflektierte diskriminierende Haltungen verändert werden sollen.

\section{Statistische Diskriminierung, ein Sonderfall?}

In der Debatte über Diskriminierung wurde von verschiedenen Seiten der Vorschlag unterbreitet, statistische und nicht-statistische Diskriminierung zu unterscheiden (Schauer 2018). So wird beispielsweise die bereits dargelegte Unterscheidung von heterosexuellen und homosexuellen Menschen in der Hämotherapierichtlinie mit der höheren Prävalenz von übertragbaren Infektionskrankheiten wie AIDS oder Hepatitis in der Gruppe der homosexuellen Männer begründet. Es gehe hierbei zum einen um das Vorliegen eines legitimen Interesses - der Eindämmung der Verbreitung übertragbarer Infektionskrankheiten - sowie zum anderen um eine statistische Korrelation der Zugehörigkeit zu einer Gruppe und dem Vorliegen einer der relevanten Infektionskrankheiten. Daher - so das Argument - fuße die Ungleichbehandlung nicht auf dem diskriminierungsrelevanten Merkmal an sich, sondern auf ebenjener erhöhten Wahrscheinlichkeit.

Statistische Korrelationen sind in der Medizin regelmäßig handlungsleitend und werden regelhaft als Rechtfertigung für Ungleichbehandlung herangezogen. Doch gilt es mit Blick auf die Gefahr der Diskriminierung immer zu prüfen, ob die Ungleichbehandlung einer Person, wegen der Zugehörigkeit zu einer statistisch erfassten Gruppe sachlich begründet ist oder nicht. Werden vermeintlich homogene Gruppeneigenschaften unterstellt (,die Homosexuellen“, „,die Muslime“ etc.), können sich leicht diskriminierende Handlungsweisen ergeben, wie der Ausschluss von homosexuellen Männern von der Blutspende eindrücklich illustriert. Das Konzept der statistischen Diskriminierung hält also an, in ethischer Hinsicht zu fragen, ob und unter welchen Umständen die aus statistischen Korrelationen gezogenen Schlussfolgerungen zur Folge haben können, einzelne Individuen als Person herabzuwürdigen oder auf andere Weise zu benachteiligen.

\section{Das Konzept der organisationalen Diskriminierung}

Die Handlungen von Personal im Gesundheitswesen werden von zahlreichen Vorschriften, z. B. Gesetzen oder Behandlungsleitlinien, gerahmt. Sie sind daher oft stark vorstrukturiert. Solche Regularien sind in Demokratien in aller Regel nicht die Produkte einzelner, sondern kollektiver Akteure, wie Regierungen, Körperschaften öffentlichen Rechts, Fachgesellschaften oder Kommissionen. Handelt ein kollektiver Akteur diskriminierend, kann dies als organisationale Diskriminierung bezeichnet werden (vgl. Altman 2020, Kap. 2.3). Im Folgenden wollen wir zeigen, welchen Mehrwert diese Differenzierung für die Medizinethik bietet.

Nicht selten tangieren Fälle organisationaler Diskriminierung Fragen der $\mathrm{Zu}$ gangsgerechtigkeit, beispielsweise wenn sich feststellen lässt, dass bestimmten Gruppen der Zugang zu medizinischen Leistungen verwehrt oder erschwert wird. 
Darüber hinaus ist diese Differenzierung für den medizinischen Kontext auch deshalb analytisch relevant, weil die Entscheidungen verschiedener kollektiver Akteure die Handlungsspielräume des Gesundheitspersonals vorstrukturieren. Dies trifft in besonderer Weise auf formelle Regelungen zu. Weisen beispielsweise Ärzt:innen oder Pflegekräfte einen homosexuellen Mann bei der Blutspende ab, so erscheint es vor dem Hintergrund der aktuellen Fassung der Hämotherapierichtlinie abwegig, die einzelnen Personen für das moralische Übel verantwortlich zu machen. Selbst wer die Zurückweisung eines homosexuellen Mannes für moralisch verwerflich hält, hat aufgrund der Regelung nur beschränkte Handlungsoptionen.

Aus ethischer Sicht wäre in solchen Fällen wichtig, ob sich Handlungsspielräume ergeben, die von den beteiligten Individuen verantwortungsvoll genutzt werden können. Das Konzept der organisationalen Diskriminierung ist insofern geeignet, die Reichweite von moralischer Verantwortung von Individuen zu präzisieren. Wie stark die Handlungsspielräume der Individuen von Regularien durch Organisationen oder Institutionen eingeschränkt werden, ist in der moralischen Beurteilung zu berücksichtigen.

\section{Wer kann diskriminiert werden?}

Für ein differenziertes Verständnis des Begriffs ist die Frage, wer diskriminiert werden kann, zentral. Hierzu lassen sich zwei Zugangsweisen identifizieren. Ein erster Zugang besteht in der Identifikation von diskriminierungsrelevanten Merkmalen. Über diese Merkmale werden Gruppen konstruiert, die von Diskriminierung betroffen sein können. Ein zweiter schreibt der Berücksichtigung von Machtverhältnissen einen besonderen Stellenwert zu. Unter dem Begriff der Intersektionalität wird kritisiert, dass abstrakt konstruierte Gruppen eine Homogenität der Lebensverhältnisse suggerieren, die es empirisch nicht gebe. Die zwei Ansätze und die intersektionale Kritik an ihnen werden wir in den folgenden Abschnitten erläutern.

\section{Diskriminierungsrelevante Merkmale und die sozial saliente Gruppe}

Ein merkmalszentriertes Verständnis liegt etwa dem rechtlichen Diskriminierungsbegriff zugrunde, ersichtlich daran, dass im Art. 3 (Abs. 3) des Grundgesetzes der Bundesrepublik Deutschland auf spezifische Merkmale abgehoben wird. Dort heißt es: „Niemand darf wegen seines Geschlechtes, seiner Abstammung, seiner Rasse, seiner Sprache, seiner Heimat und Herkunft, seines Glaubens, seiner religiösen oder politischen Anschauungen benachteiligt oder bevorzugt werden. Niemand darf wegen seiner Behinderung benachteiligt werden." Alle gelisteten Merkmale eint, dass sie als Merkmale sozialer Gruppen zu verstehen sind. Lippert-Rasmussen (2006, 2013) zufolge können es nicht beliebige Merkmale sein, die solche Gruppen ausmachen. Ausschlaggebend sei, dass es sozial salient sei. ${ }^{6}$ Charakteristisch für ein sozial salientes Merkmal ist, dass es Interaktionen situationsübergreifend strukturiert

\footnotetext{
${ }^{6}$ Einen Überblick zu weiteren Auffassungen dazu, wie diskriminierungsrelevante Merkmale verstanden und Gruppen definiert werden können, gibt Heinrichs (2015, S. 27).
} 
(Lippert-Rasmussen 2006, S. 169). Dies gilt dann auch für die unter Umständen herabsetzenden Stereotype, die mit dem Merkmal und der entsprechenden Gruppe verbunden sind, wie dies etwa bei Menschen mit Behinderung, mit Fluchterfahrung oder sehr dicken Menschen oft der Fall ist.

Mit dem Konzept der socially salient group wird also auf eine Tendenz zur lebensbereichs- und situationsübergreifenden Wiederholung von diskriminierenden Erfahrungen verwiesen (vgl. Lippert-Rasmussen 2013, S. 34). Typischerweise haben Personen, die einer socially salient group zugerechnet werden, eine geteilte Historie und teilen überdies Erfahrungen im aktuellen Gefüge der sozialen Verhältnisse.

Ein Beispiel aus dem medizinischen Kontext: Eine Ärztin führt, begleitet von einem Pfleger, die Visite auf einer Station im Krankenhaus durch. In den Krankenzimmern wird die Ärztin jedoch wiederholt als „Schwester“ und der begleitende Pfleger als „Herr Doktor“ angesprochen. Diese Situation lässt sich mithilfe des Konzepts der socially salient group einordnen. Die Patient:innen nehmen die Ärztin als Mitglied der sozial salienten Gruppe der Frauen wahr und verorten sie auf dieser Basis stereotyp in einer helfenden Berufsgruppe innerhalb des hierarchisch organisierten Berufsfelds der medizinischen Professionen. Es kann davon ausgegangen werden, dass die Ärztin in ihrem Leben wiederkehrend solche Situationen erlebt, in denen ihre Expertise nicht wahrgenommen und ihr Status nicht anerkannt wird.

Die Idee der socially salient group ist weithin akzeptiert, wie der oben genannte Grundgesetzartikel zeigt. Zwei wesentliche Kritikpunkte bringt Thomsen (2013) vor. Er vertritt die Ansicht, dass ein überzeugendes Gruppenkriterium an eine Schadensschwelle gebunden sein müsse und es zudem zirkulär sei. Es sei nur dann überzeugend, wenn es für jeden hypothetischen Fall erklären könne, weshalb eine Ungleichbehandlung aufgrund der Zugehörigkeit zu einer sozial salienten Gruppe moralisch schwerer wiege, als wenn sie wegen eines anderen Merkmals, wie z.B. der Augenfarbe, geschehe. Hierfür müsse eine Schwelle identifizierbar sein, ab welcher der zusätzliche Schaden, der durch die situationsübergreifende Wiederholung diskriminierender Handlungen entsteht, moralisch schwerwiegender sei (Thomsen 2013, S. 128-140). Dabei lässt er jedoch außer Acht, dass nicht nur durch die faktische Wiederholung ein Schaden entsteht. Ein gutes Leben lässt sich auch als eines interpretieren, das frei von der begründeten Angst vor (sich wiederholender) herabwürdigender, schädigender oder die Chancengleichheit beeinträchtigender Ungleichbehandlung ist. Die Erwartbarkeit der Wiederholung, mit welcher die Mitglieder einer von Diskriminierung betroffenen sozial salienten Gruppe leben müssen, kann bereits eigene schädigende Effekte haben. Eine bekannte Problematik ist etwa, dass Mitglieder von Gruppen, die Diskriminierung im Gesundheitswesen befürchten, nicht selten medizinische Einrichtungen meiden und deshalb einen schlechteren gesundheitlichen Allgemeinzustand aufweisen.

Problematisch ist zudem, dass die soziale Salienz eines Merkmals sowohl von Kritikern wie Thomsen als auch von Lippert-Rasmussen oder Heinrichs (2015, S. 27) essentialistisch verstanden wird. Sie setzen voraus, dass die soziale Salienz einem Merkmal wie z.B. dem Geschlecht innewohnt. Demgegenüber kann man aus einer konstruktivistischen Sichtweise argumentieren, dass die lebensbereichsübergreifende Bedeutung eines Merkmals größtenteils sozial hergestellt wird. Deshalb halten die Soziolog:innen Lemke und Liebsch im Kontext genetischer Diskriminierung 
dazu an, „die soziale Konstruktion von Normen und Unterscheidungen selbst zum Gegenstand der Analyse zu machen“ (Lemke und Liebsch 2015, S. 172).

Aus einer soziologischen Perspektive wird es als relevant bewertet, welche Bedeutungen machtvolle gesellschaftliche Akteure wie die Medizin einem Merkmal zuschreiben. Sie haben einen diskursprägenden Einfluss im Sinne einer foucaultschen „Biomacht“. Allgemein lässt sich formulieren, dass die Zuschreibungen der Medizin dann problematisch sind, wenn sie pauschal einer Ausprägung menschlicher Vielfalt einen Krankheitswert zuweist, sie somit als „defizitär“ stigmatisiert und moralisch auflädt. Da die Medizin ein hohes gesellschaftliches Ansehen genießt und breite Akzeptanz erfährt, nimmt sie erheblichen Einfluss darauf, wer in einer Gesellschaft als normal und wer als ,in unerwünschter Weise anders“ (Goffman 1975, S. 13) gilt. Dies wird im Zusammenhang mit einer Reihe von Merkmalen, etwa Behinderung als ungerechtfertigte „Pathologisierung“ kritisiert (vgl. Schidel 2020).

Es ergibt sich so eine wechselseitige Bedingtheit von diskriminierungsrelevanten Merkmalen und sozialen Herstellungsprozessen (Vehmas und Watson 2014). Dies erhöht die Komplexität der ethischen Problematik unweigerlich, da es die Frage nach der Verantwortung der Medizin für den Erhalt und die Produktion der sozialen Bedeutung eines Merkmals aufwirft, insbesondere da diese über die Grenzen des Gesundheitswesens hinaus ihre Wirksamkeit entfalten.

\section{Sozialer Status und Macht}

Hellman geht davon aus, dass die Frage, wer diskriminiert werden kann, mit einem merkmalsbezogenen Ansatz nicht abschließend beantwortet werden kann (Hellman 2018, S. 100). Deshalb schlägt sie vor, den sozialen Status der involvierten Akteure einzubeziehen und rückt die Analyse von Machtverhältnissen in den Fokus.

Machtunterschiede sind in der Medizin omnipräsent. Manche sind leicht ersichtlich, weil sie durch Regelwerke festgelegt sind (z. B. in hierarchischen Berufsordnungen). Dies sind jedoch nicht die einzigen Machtunterschiede, die in einer ethischen Debatte über Diskriminierung im Gesundheitswesen zu berücksichtigen sind. Auch Machtunterschiede, wie etwa zwischen den Geschlechtern oder zwischen People of Color und Weißen Menschen, die gesamtgesellschaftliche Relevanz haben, zeigen im Gesundheitswesen Auswirkung und strukturieren die Interaktionen zwischen Personal und Patient:innen.

Wie schon dargestellt, sieht Hellman das zentrale Übel von Diskriminierung in der Herabwürdigung des Individuums. Dazu bedürfe es einer gewissen Machtposition (Hellman 2018, S. 103). Nach Hellman verfügt eine Person mit „sufficient power“ über die Macht, mit ihren Worten oder Handlungen eine andere Person auf herabwürdigende Weise zu treffen. Am einfachsten lässt sich der soziale Status einer Person nachvollziehen, wenn formelle Regeln wie Gesetze die Rollen der Interaktionspartner:innen definieren. Hierzu ein Beispiel. Möchte eine transgeschlechtliche Person in Deutschland ihren Personenstand ändern, so ist dies an zwei positive psychiatrische Gutachten gebunden. Fallen die Gutachten negativ aus, muss sie weiterhin mit einem amtlichen Geschlechtseintrag leben, der nicht ihrer Geschlechtsidentität entspricht. Die Gutachtenden sind also qua Gesetz in einer machtvollen Position, über 
die geschlechtliche Identität der begutachteten Person zu entscheiden. Sie haben damit auch die Macht, diese Person herabzuwürdigen.

Andere Autoren sehen in Machtasymmetrie hingegen keine notwendige Bedingung für Diskriminierung (Blum 2002; Lippert-Rasmussen 2006, S. 173). Blum (2002) geht es hierbei nicht um eine prinzipielle Entkräftung der Bedeutung von Macht, sondern um eine Differenzierung. Er stellt fest, dass sich an ihr lediglich die „moral seriousness“ (Blum 2002, S. 39) einer Handlung bewerten lasse.

Das Gesundheitswesen ist ein Ort, an dem organisationsbedingte und gesellschaftliche Machtunterschiede gleichzeitig wirken und sich gegenseitig beeinflussen. Diese Verschränkung verleiht der Thematik eine zusätzliche Brisanz und sollte in einer differenzierten Debatte über Diskriminierung in der Medizin nicht ausgeklammert werden.

\section{Intersektionalität}

Aus intersektionaler Perspektive wird die Eindimensionalität der oben genannten Ansätze kritisiert. Crenshaw (1989) argumentierte, dass die Mitglieder der Gruppe der „Frauen“ wegen der Zugehörigkeit zu weiteren sozial salienten Gruppen (z. B. „Schwarze“ o. „Weiße“) oder entlang von anderen Ungleichheitsdimensionen, wie dem soziö̈konomischen Hintergrund, sehr unterschiedliche gesellschaftliche Erfahrungen machen. Manche Kombinationen können verstärkend, andere entschärfend wirken. Im Kontext genetischer Diskriminierung fordern daher auch Lemke und Liebsch zu untersuchen, ,wie sich Formen genetischer Diskriminierung mit sexistischen und rassistischen Praktiken verbinden und diese ggf. wechselseitig verstärken“ (2015, S. 172).

Die Bedeutung des intersektionalen Blicks zeigt sich etwa an folgendem Beispiel: Eine Gynäkologin in Deutschland, die sich durch ihr Kopftuch als Muslima zu erkennen gibt, erfährt regelmäßig, dass ihre Expertise von Kolleg:innen nicht anerkannt wird. Aus intersektionaler Perspektive wird ihre soziale Position, etwa in einem Konsiliargespräch mit Kolleg:innen, von beiden Faktoren bestimmt. Die Kolleg:innen nehmen sie als Mitglied der Gruppe der Frauen und der Personen muslimischen Glaubens wahr. So ist also denkbar, dass ihr wissenschaftlicher Rat nicht nur wegen ihrer Zuordnung zur Gruppe der Frauen weniger ernst genommen wird, sondern dass er auch reflexhaft als einem traditionalistischen Frauenbild geschuldet angesehen wird, das vermeintlich mit dem Tragen eines Kopftuchs ${ }^{7}$ zum Ausdruck gebracht werde. Wichtig ist, dass es dabei nicht um Addition von negativ konnotierten Eigenschaften verschiedener Gruppen geht, sondern um die spezifische Position eines Individuums. Phänomene wie Sexismus, Rassismus oder Ableism sind nicht nebeneinander, sondern als miteinander verschränkt zu verstehen. Festzuhalten ist, dass die intersektionale Kritik Kernideen wie den Wiederholungscharakter von Diskriminierung nicht entkräftet; es werden so nur die Grenzen eindimensionaler Ansätze ausgewiesen (vgl. Stoljar 2018, S. 77).

\footnotetext{
7 Für einen Einblick in Argumentationen iranischer Frauenrechtlerinnen siehe Armipur (2003), für eine Studie zu Bedeutungen des muslimischen Kopftuchs siehe Sahin (2014).
} 


\section{Schlussfolgerungen}

In diesem Kapitel haben wir zwei unterschiedliche Zugänge zur Frage, wer diskriminiert werden kann, beleuchtet. Wenngleich jeder eigene Aspekte betont, kommen sie beide zu dem Schluss, dass nicht jede beliebige Person, sondern nur bestimmte Personen von Diskriminierung betroffen sein können. Diese lassen sich als Mitglieder sozial salienter Gruppen verstehen, die über wenig soziale Macht verfügen und deshalb eine nachrangige soziale Position in der Gesellschaft einnehmen. Das Konzept der Intersektionalität hält dazu an, die situationsstrukturierende Bedeutung von Gruppenzugehörigkeiten im Lichte einander mal verstärkender, mal aufhebender Prozesse zu betrachten, um so ein differenzierteres Bild zu erhalten.

Unseres Erachtens stehen die vorgestellten Ansätze nicht im Widerspruch zueinander, sondern sind gemeinsam unentbehrlich, um zu verstehen, was Diskriminierung charakterisiert. Es ist die Erwartbarkeit der Wiederholung der herabsetzenden oder anderweitig schädigenden Verhaltensweisen, die einen signifikant nachteiligen Einfluss auf Selbstbild und Lebensweise der betroffenen Personen hat. Sie verhindert ein Leben frei von Angst vor sich wiederholender herabwürdigender, schädigender oder die Chancengleichheit beeinträchtigender Ungleichbehandlung. Wesentlich ist, sozial saliente Merkmale nicht essentialistisch zu verstehen, sondern als Ergebnis der Wechselwirkung biologischer und sozialer Diskurse. Die Medizin als machtvolle gesellschaftliche Instanz ist daran beteiligt, Merkmalen eine Bedeutung und gesellschaftlichen Gruppen somit überhaupt erst soziale Salienz zu verleihen. Sind diese Bedeutungen negativ, verfestigt dies ihre nachrangige gesellschaftliche Position im Gefüge sozialer Machtverhältnisse nachhaltig.

\section{Epistemische Ungerechtigkeit}

Die Medizin ist ein wissensbasiertes System von großer gesellschaftlicher Bedeutung. Wer im Namen der Medizin spricht, kann in der Regel nicht nur wissenschaftliche, sondern auch gesellschaftliche Autorität beanspruchen. Explizite und implizite Wissensordnungen haben einen Einfluss darauf, wer in einer Gesellschaft gehört wird, wessen Stimme zählt, wer Glaubwürdigkeit beanspruchen kann und wer nicht. Machtunterschiede und soziale Stereotype beeinflussen die Position der Sprechenden im Gesundheitswesen und verzerren den Erkenntnisprozess. Dies kann nach Miranda Fricker (2007) als epistemische Ungerechtigkeit bezeichnet werden. Manche Autor:innen sehen darin eine eigene Form der Diskriminierung, die sogenannte epistemische Diskriminierung (vgl. Puddifoot 2018), weshalb hier näher darauf eingegangen werden soll.

Fricker unterscheidet im Wesentlichen zwei Formen epistemischer Ungerechtigkeit: „testimonial injustice“ (Fricker 2007, Kap. 2) und „hermeneutical injustice“ (Fricker 2007, Kap. 7). „Testimonial injustice“ erfährt eine Person, wenn ihre Glaubwürdigkeit aufgrund ihrer sozialen Gruppenzugehörigkeit herabgewürdigt wird. Ihre Positionen werden dann nicht gehört, relativiert oder nicht ernst genommen. Dies ist moralisch bedenklich, weil ihr pauschal die Fähigkeit abgesprochen wird, Wissen zu generieren und es an andere weitergeben zu können. Sie wird, in den Worten 
Frickers, herabgewürdigt als Wissende und damit auch als Mensch (Fricker 2007, S. 44). Solche Situationen treten insbesondere dann gehäuft auf, wenn sich die Zuhörenden von stereotypen Verallgemeinerungen über den epistemischen Status der Mitglieder einer sozialen Gruppe leiten lassen. Ein Beispiel ist die anamnestische Untersuchung eines erkrankten zehnjährigen Kindes, das von seinen Eltern begleitet wird, und bei dem ausschließlich mit den Eltern kommuniziert wird. Eltern gelten bei Kinderkrankheiten zwar nicht zu Unrecht als zuverlässigere Quelle für Informationen als das erkrankte Kind, werden dessen Ansichten wegen seiner Zugehörigkeit zur Gruppe der Kinder jedoch pauschal als irrelevant behandelt, widerfährt ihm ,,testimonial injustice“.

Wichtig ist, dass ,testimonial injustice“ genauso wie Diskriminierung durch wiederholtes und lebensbereichsübergreifendes Auftreten charakterisiert ist. Die wohl erheblichste Langzeitfolge kann darin bestehen, dass die Betroffenen den Glauben an sich selbst, ihre intellektuellen Fähigkeiten und ihre Entwicklungspotenziale verlieren (Fricker 2007, S. 58).

Wird die Glaubwürdigkeit von den Mitgliedern einer sozial salienten Gruppen aufgrund der Deutungshoheit dominanter gesellschaftlicher Gruppen oder Akteure dauerhaft herabgewürdigt, kann dies dazu führen, dass es dem gesamten Kollektiv an Begriffen fehlt, um negative Erfahrungen als solche deuten zu können (Fricker 2007, S. 159). Ihnen widerfährt dann ,hermeneutical injustice“ (Fricker 2007, Kap. 7). Dies lässt sich beispielhaft an der Situation intergeschlechtlicher Personen illustrieren, die unter den Folgen geschlechtlich vereindeutigender Operationen in der Kindheit leiden. Über einen langen Zeitraum hinweg wurden diese Maßnahmen als alternativlos und medizinisch notwendig dargestellt und durchgeführt. Aufgrund der Deutungshoheit der Medizin mangelte es den Betroffenen an Konzepten, um ihre negativen Erfahrungen bei diesem Vorgehen einordnen, kommunizieren und letztlich auch kritisieren zu können.

Frickers Konzept von epistemischer Ungerechtigkeit ist geeignet, um ein Schädigungspotenzial von Diskriminierung aufzuzeigen, das für den Kontext der Medizin als Institution von hohem gesellschaftlichem Rang von besonderer Bedeutung ist. Die dadurch erzeugte Macht-Asymmetrie beeinflusst nicht nur das konkrete Gespräch zwischen Ärzt:in und Patient:in, sondern ganz allgemein, ob und wie die Sichtweise betroffener Personen wahrgenommen und respektiert wird. Für diese Gefahren sensibel zu sein, ist für die Mitarbeitenden im Gesundheitswesen wichtig, weil nur so alle für den Erkenntnisprozess relevanten Stimmen gehört werden können.

\section{Zusammenfassende Überlegungen}

Diskriminierung ist ein Konzept, das sich mit Gewinn auf die Medizin anwenden lässt. Für die praktischen Belange des Gesundheitswesens ist unseres Erachtens ein pluralistisches Verständnis angemessen. Diskriminierung ist demnach moralisch relevant, weil sie Individuen schädigt, ihre Freiheitsrechte beschneidet, sie herabwürdigt oder ihre Chancengleichheit beeinträchtigt. Schädigende Effekte gehen dabei schon von der Erwartbarkeit der Wiederholung aus, mit welcher die Mitglieder einer 
von Diskriminierung betroffenen sozial salienten Gruppe leben müssen. Wer etwa im Gesundheitswesen nicht frei ist von der Angst vor sich wiederholender herabwürdigender Behandlung, wird ggf. allein schon deshalb den Kontakt zu Ärzt:innen meiden. Da es sich bei der Medizin um ein wissensbasiertes System handelt, müssen zudem Formen epistemischer Benachteiligung (,Nicht-gehört-Werden“ oder „Nichternst-genommen-Werden“) berücksichtigt werden.

Diskriminiert werden können nur Mitglieder sozial salienter Gruppen mit einem niedrigeren Status im Gefüge sozialer Machtverhältnisse. Die situationsübergreifende Bedeutung des Merkmals wird überwiegend sozial hergestellt. Als machtvoller gesellschaftlicher Akteur ist die Medizin in der Lage, auf solche Prozesse in negativer wie positiver Hinsicht nachhaltig Einfluss zu nehmen, etwa darauf, wer in einer Gesellschaft als normal und wer als in unerwünschter Weise anders gilt.

Es ist jedoch zu berücksichtigen, dass jeder Mensch unterschiedlichen sozialen Gruppen zugerechnet werden kann. Sie alle beeinflussen, welche soziale Position ein Individuum, etwa in einem Gespräch, einnimmt.

Die Unterscheidung von direkter und indirekter Diskriminierung hilft insbesondere dabei, weniger offensichtliche Formen zu erkennen. Direkte Diskriminierung, also die offensichtlich unbegründete Ungleichbehandlung von Menschen entlang eines sozial salienten Merkmals wie etwa „Hautfarbe“, ist die in der Regel besser bekannte Problematik und wird im Gesundheitswesen mittlerweile vermutlich eher selten sein. Indirekte Diskriminierung auf der Basis scheinbar neutraler Kriterien ist dagegen wesentlich schwerer zu identifizieren und gerade im Gesundheitswesen nicht unüblich. Insbesondere der Einsatz von statistischen Korrelationen in Bezug auf sozial saliente Gruppen ist vor diesem Hintergrund auf diskriminierende Effekte zu hinterfragen.

Absicht ist keine notwendige Voraussetzung für Diskriminierung. Der Bezug auf die Absicht der handelnden Person ist jedoch wichtig, um die jeweils geforderte spezifische Form von Verantwortung zu bestimmen. Im Kontext der Auseinandersetzung mit Diskriminierung im Gesundheitswesen spielt dies eine große Rolle, denn die einzelnen professionellen Individuen handeln gemeinhin zwar in wohlwollender Absicht, aber dennoch mit mitunter diskriminierenden Auswirkungen. Die moralische Verantwortung besteht darin, für solche Formen sensibel zu sein und ihrem Auftreten möglichst vorzubeugen. Insbesondere in komplexen organisatorischen Gefügen wie dem Gesundheitswesen sind Formen organisationaler Diskriminierung häufiger anzutreffen. Institutionelle und rechtliche Vorgaben schränken den Handlungsspielraum und damit auch das Ausmaß der moralischen Verantwortung der Akteure (Ärzt:innen, Pflegekräfte etc.) ein. Dennoch tragen die individuell handelnden Personen eine gewisse Verantwortung, den jeweiligen Status der Institution oder Organisation zu reflektieren und ggf. nach Auswegen für ein ethisch verantwortliches Vorgehen zu suchen.

Die Erkenntnisse der sozialpsychologischen und soziologischen Forschung erlauben es, die zugrundeliegenden sozialen Mechanismen besser zu verstehen und die vielschichtigen Ursachen von Diskriminierung in einer Gesellschaft zu erfassen. Als wissensbasiertes System von großem gesellschaftlichen Einfluss weist das Gesundheitswesen eine Reihe von Eigenschaften auf, die Diskriminierung begünstigen 
können. Dies zu erkennen ist unerlässlich, um Diskriminierung wirksam entgegentreten zu können.

Förderung Die Forschungen zu diesem Beitrag fanden statt im Rahmen des interdisziplinären Projekts TRANS*KIDS - einem Projekt zur Förderung eines nicht-diskriminierenden Umgangs mit jungen trans*Personen durch patientenorientierte Schulungsmaßnahmen im Gesundheitswesen, gefördert durch das Bundesministerium für Gesundheit (Projektleitung: G. Romer, Universität Münster, https://transkidsstudie.de/).

Funding Open Access funding enabled and organized by Projekt DEAL.

Open Access Dieser Artikel wird unter der Creative Commons Namensnennung 4.0 International Lizenz veröffentlicht, welche die Nutzung, Vervielfältigung, Bearbeitung, Verbreitung und Wiedergabe in jeglichem Medium und Format erlaubt, sofern Sie den/die ursprünglichen Autor(en) und die Quelle ordnungsgemäß nennen, einen Link zur Creative Commons Lizenz beifügen und angeben, ob Änderungen vorgenommen wurden.

Die in diesem Artikel enthaltenen Bilder und sonstiges Drittmaterial unterliegen ebenfalls der genannten Creative Commons Lizenz, sofern sich aus der Abbildungslegende nichts anderes ergibt. Sofern das betreffende Material nicht unter der genannten Creative Commons Lizenz steht und die betreffende Handlung nicht nach gesetzlichen Vorschriften erlaubt ist, ist für die oben aufgeführten Weiterverwendungen des Materials die Einwilligung des jeweiligen Rechteinhabers einzuholen.

Weitere Details zur Lizenz entnehmen Sie bitte der Lizenzinformation auf http://creativecommons.org/ licenses/by/4.0/deed.de.

\section{Einhaltung ethischer Richtlinien}

Interessenkonflikt M. Hädicke und C. Wiesemann geben an, dass kein Interessenkonflikt besteht.

Ethische Standards Für diesen Beitrag wurden von den Autorinnen keine Studien an Menschen oder Tieren durchgeführt. Für die aufgeführten Studien gelten die jeweils dort angegebenen ethischen Richtlinien.

\section{Literatur}

Altman A (2020) Discrimination. In: Zalta E (Hrsg) Encyclopedia of philosophy. Metaphysics Research Lab, Stanford University. https://plato.stanford.edu/archives/win2020/entries/discrimination/. Zugegriffen: 28. Okt. 2020

Antidiskriminierungsstelle des Bundes (2017) Dritter Gemeinsamer Bericht der Antidiskriminierungsstelle des Bundes und der in ihrem Zuständigkeitsbereich betroffenen Beauftragten der Bundesregierung und des Deutschen Bundestages. https://www.antidiskriminierungsstelle.de/SharedDocs/Downloads/ DE/publikationen/BT_Bericht/Gemeinsamer_Bericht_dritter_2017.pdf. Zugegriffen: 28. Okt. 2020

Armipur K (2003) Emanzipation trotz Kopftuch - Iranische FrauenrechtlerInnen streiten um die Deutung des Korans. Fem Stud 21(2):213-225. https://doi.org/10.1515/fs-2003-0204

Beeghly E (2018) Discrimination and disrespect. In: Lippert-Rasmussen K (Hrsg) The Routledge handbook of the ethics of discrimination. Routledge, Abingdon, S 83-96

Blum L (2002) "I'm not a racist, but..." The moral quandary of race. Cornell University Press, Ithaca

Boshammer S (2008) Diskriminierung. In: Gosepath S, Rössler B, Hinsch W (Hrsg) Handbuch der Politischen Philosophie und Sozialphilosophie. De Gruyter, Berlin, S 232-238 (2 Bände)

Brownstein S (2019) Implicit bias. In: Zalta E (Hrsg) Encyclopedia of philosophy. Metaphysics Research Lab, Stanford University. https://plato.stanford.edu/entries/implicit-bias/. Zugegriffen: 15. Jan. 2021

Bundesärztekammer (BÄK) (2017) Richtlinie zur Gewinnung von Blut und Blutbestandteilen und zur Anwendung von Blutprodukten (Richtlinie Hämotherapie). Gesamtnovelle. https://www.bundesaerzte kammer.de/fileadmin/user_upload/downloads/pdf-Ordner/MuE/Richtlinie_Haemotherapie_E_A_ 2019.pdf. Zugegriffen: 28. Okt. 2020 
Bundesärztekammer (BÄK) (2006) (Muster-)Richtlinie zur Durchführung der assistierten Reproduktion Novelle 2006. Dtsch Arztebl 103(20):A 1394-A 1398

Crenshaw K (1989) Demarginalizing the intersection of race and sex: a black feminist critique of antidiscrimination doctrine. Univ Chic Leg Forum 140:139-167

Deutsche Aidshilfe (2020) Blutspendeverbot für schwule und bisexuelle Männer. Positionen. https://www. aidshilfe.de/blutspendeverbot-schwule-bisexuelle-maenner. Zugegriffen: 28. Okt. 2020

Deutsche Interdisziplinäre Vereinigung für Intensiv- und Notfallmedizin (DIVI) (2020) Entscheidungen über die Zuteilung intensivmedizinischer Ressourcen im Kontext der COVID-19-Pandemie. Version 2. Klinisch-ethische Empfehlungen. https://www.divi.de/empfehlungen/publikationen/covid19-ethik-empfehlung-v2/download. Zugegriffen: 25. Jan. 2021

Eidelson B (2015) Discrimination and disrespect. Oxford University Press, Oxford

Fitzgerald C, Hurst S (2017) Implicit bias in healthcare professionals: a systematic review. BMC Med Ethics. https://doi.org/10.1186/s12910-017-0179-8

Fricker M (2007) Epistemic injustice: power and ethics of knowing. Oxford University Press, New York

Gen-ethisches Netzwerk e. V. (2014) Pränataldiagnostik fördert Diskriminierung! Blogeintrag. https://genethisches-netzwerk.de/maerz-2014/praenataldiagnostik-foerdert-diskriminierung. Zugegriffen: 27. Okt. 2020

Goffman E (1975) Stigma. Über Techniken der Bewältigung einer beschädigten Identität. Suhrkamp, Frankfurt a. M.

Graumann S, Koopmann L (2018) Neue Entwicklungen in der pränatalen Diagnostik - gesellschaftliche und ethische Fragen. https://kidoks.bsz-bw.de/frontdoor/deliver/index/docId/1352/file/PND+Werk stattbericht+Graumann+Koopmann.pdf. Zugegriffen: 25. Jan. 2021 (Bochum: Ev. Hochschule Rheinland-Westfalen-Lippe)

Heinrichs B (2015) Diskriminierung. In: Sturma D, Heinrichs B (Hrsg) Handbuch Bioethik. Metzler, Stuttgart, S 26-31

Hellman D (2008) When is discrimination wrong? Harvard University Press, Cambridge

Hellman D (2018) Discrimination and social meaning. In: Lippert-Rasmussen K (Hrsg) The Routledge handbook of the ethics of discrimination. Routledge, Abingdon, S 97-107

Klonschinski A (2020) Einleitung: Was ist Diskriminierung und was genau ist daran moralisch falsch? Z Prakt Philos 7(1):133-154. https://doi.org/10.22613/zfpp/7.1.5

Knight C (2018) Discrimination and equality of opportunity. In: Lippert-Rasmussen K (Hrsg) The Routledge handbook of the ethics of discrimination. Routledge, Abingdon, S 140-149

Lemke T, Liebsch K (Hrsg) (2015) Die Regierung der Gene. Diskriminierung und Verantwortung im Kontext genetischen Wissens. Springer VS, Wiesbaden

Lippert-Rasmussen K (2006) The badness of discrimination. Ethical Theory Moral Pract 9:167-185. https://doi.org/10.1007/s10677-006-9014-x

Lippert-Rasmussen K (2013) Born free and equal? A philosophical inquiry into the nature of discrimination. Oxford University Press, Oxford

Lippert-Rasmussen K (2018) The philosophy of discrimination: an introduction. In: Lippert-Rasmussen K (Hrsg) The Routledge handbook of the ethics of discrimination. Routledge, Abingdon, S 1-16

Loss J, Nagel E (2009) Probleme und ethische Herausforderungen bei der bevölkerungsbezogenen Gesundheitskommunikation. Bundesgesundheitsblatt Gesundheitsforschung Gesundheitsschutz 52(5):502-511. https://doi.org/10.1007/s00103-009-0839-z

Merkel R (2019) Von wegen Selektion. Feuilletonbeitrag. https:/www.faz.net/aktuell/feuilleton/debatten/ diskriminiert-ein-bluttest-embryonen-mit-trisomie-21-16157312.html. Zugegriffen: 27. Okt. 2020

Moreau S (2010) What is discrimination? Philos Public Aff 38(2):143-179

Mukwende M, Tamony P, Turner M (2020) Mind the gap.-A handbook of clinical signs in black and brown skin. St. George's University of London. https://www.blackandbrownskin.co.uk/mindthegap. Zugegriffen: 25. Jan. 2021

Nationale Akademie der Wissenschaften, Union der Akademien der Wissenschaften (2019) Fortpflanzungsmedizin in Deutschland - für eine zeitgemäße Gesetzgebung. Stellungnahme. https://www. leopoldina.org/uploads/tx_leopublication/2019_Stellungnahme_Fortpflanzungsmedizin_web_01. pdf. Zugegriffen: 28. Okt. 2020

Obermeyer Z, Powers B, Vogeli C, Mullainathan S (2019) Dissecting racial bias in an algorithm used to manage the health of populations. Science 366:447-453. https://doi.org/10.1126/science.aax2342

Puddifoot K (2018) Epistemic discrimination. In: Lippert-Rasmussen K (Hrsg) The Routledge handbook of the ethics of discrimination. Routledge, Abingdon, S 54-67 
Ray K (2020) Black bioethics and how the failures of the profession paved the way for its existence. Blogeintrag. http://www.bioethics.net/2020/08/black-bioethics-and-how-the-failures-of-theprofession-paved-the-way-for-its-existence/. Zugegriffen: 27. Okt. 2020

Rehmann-Sutter C (2005) Die Ungerechtigkeit genetischer Diskriminierung. In: Rehmann-Sutter C (Hrsg) Zwischen den Molekülen: Beiträge zur Philosophie der Genetik. Francke, Tübingen, S 269-281

Sahin R (2014) Die Bedeutung des muslimischen Kopftuchs: Eine kleidungssemiotische Untersuchung Kopftuch tragender Musliminnen in der Bundesrepublik Deutschland. LIT Verlag, Münster

Scanlon T (2008) Moral dimensions: permissibility, meaning, blame. Harvard University Press, Cambridge

Schaber P (2012) Menschenwürde: ein für die Medizinethik irrelevanter Begriff? Ethik Med 24:297-306. https://doi.org/10.1007/s00481-012-0218-4

Schauer F (2018) Statistical (and non-statistical) discrimination. In: Lippert-Rasmussen K (Hrsg) The Routledge handbook of the ethics of discrimination. Routledge, Abingdon, S 42-53

Schidel R (2020) Pränataldiagnostik als Instanz von struktureller Diskriminierung? Überlegungen zur Debatte um den PraenaTest und seine Auswirkungen auf Menschen mit Behinderung. Z Prakt Philos 7(1):231-264. https://doi.org/10.22613/zfpp/7.1.8

Steger F (Hrsg) (2007) Was ist krank? Stigmatisierung und Diskriminierung in der Medizin und Psychotherapie. Psychosozial-Verlag, Gießen

Stoecker R (2011) Wozu brauchen wir in der medizinischen Ethik die Menschenwürde? In: Joerden J, Hilgendorf E, Petrillo N, Thiele F (Hrsg) Menschenwürde und moderne Medizintechnik. Nomos, Baden-Baden, S 197-213

Stoljar N (2018) Discrimination and intersectionality. In: Lippert-Rasmussen K (Hrsg) The Routledge handbook of the ethics of discrimination. Routledge, Abingdon, S 287-297

Thomsen F (2011) We will find the black man who did this. Conceptual and ethical studies of discrimination in criminal justice. Dissertation, Roskilde University

Thomsen F (2013) But some groups are more equal than others: a critical review of the group-criterion in the concept of discrimination. Soc Theory Pract 39(1):120-146

Thomsen F (2015) Stealing bread and sleeping beneath bridges-indirect discrimination as disadvantageous equal treatment. Moral Philos Politics 2:299-327. https://doi.org/10.1515/mopp-2014-0027

United Nations Educational, Scientific and Cultural Organization (UNESCO) (2005) Universal declaration on bioethics. http://portal.unesco.org/en/ev.php-URL_ID=31058\&URL_DO=DO_TOPIC\& URL_SECTION=201.html. Zugegriffen: 27. Okt. 2020

Vehmas S, Watson N (2014) Moral wrongs, disadvantages, and disability: a critique of critical disability studies. Disabil Soc 29(4):638-650. https://doi.org/10.1080/09687599.2013.831751

Verein für Gleichstellung und Menschenrechte Behinderter e. V. (NW3), Interessenvertretung Selbstbestimmt Leben in Deutschland e. V. (ISL) (2020) Triage - Behinderung darf kein Kriterium bei Priorisierungs-Entscheidungen sein! Kommentar zu den klinisch-ethischen Empfehlungen „Entscheidungen über die Zuteilung von Ressourcen..." der Fachgesellschaften vom 25. März 2020. http://nw3.de/ attachments/article/136/Kommentar\%20zu\%20Triage.pdf. Zugegriffen: 25. Jan. 2021 